\title{
ON THE ENERGY OF A LARGE ATOM
}

\section{CHARLES L. FEFFERMAN AND LUIS A. SECO}

We announce a proof of an asymptotic formula for the groundstate energy of a large atom. The early work of Thomas-Fermi, Hartree-Fock, Dirac, and Scott predicted that for an atomic number $Z$, the energy is $E(Z) \approx-c_{0} Z^{7 / 3}+c_{1} Z^{2}-c_{2} Z^{5 / 3}$ for known $c_{0}, c_{1}$, and $c_{2}$ (see [5]). Schwinger [7] observed an additional effect and set down the modified formula $E(Z) \approx-c_{0} Z^{7 / 3}+c_{1} Z^{2}-$ $\frac{10}{9} c_{2} Z^{5 / 3}$. Our proof shows that Schwinger's formula is correct.

We give the precise formulation of the problem. For a fixed nucleus of charge $Z$ and quantized electrons $x_{1}, \ldots, x_{N} \in \mathbf{R}^{3}$, the Hamiltonian $H_{N, Z}$ is the self-adjoint operator

$$
\sum_{k=1}^{N}\left(-\Delta_{x_{k}}-\frac{Z}{\left|x_{k}\right|}\right)+\frac{1}{2} \sum_{i \neq j} \frac{1}{\left|x_{i}-x_{j}\right|} .
$$

This operator acts on functions $\psi\left(x_{1}, \ldots, x_{N}\right)$ which satisfy the antisymmetry condition

$$
\psi\left(x_{1}, \ldots, x_{N}\right)=(\operatorname{sgn} \sigma) \psi\left(x_{\sigma 1}, \ldots, x_{\sigma N}\right)
$$

for permutations $\sigma$. The ground-state energy $E(N, Z)$ is defined as the infimum of the spectrum of $H_{Z, N}$, and the ground-state energy of an atom is defined as $E(Z)=\inf _{N \geq 0} E(N, Z)$. We have ignored electron spin, which simplifies notation, alters no ideas, and causes some of our coefficients to differ from those in the physics literature. Our main result is as follows.

Theorem. $E(Z)=-c_{0} Z^{7 / 3}+\frac{1}{8} Z^{2}-\frac{10}{9} c_{2} Z^{5 / 3}+O\left(Z^{(5 / 3)-a}\right)$ with $a>0$, and $c_{0}, c_{2}$ to be described below.

Hughes [1] and Siedentop-Weikard [9] recently proved the "Scott conjecture," i.e. $E(Z)=-c_{0} Z^{7 / 3}+\frac{1}{8} Z^{2}+O\left(Z^{2-a}\right)$ with $a=1 / 24$. (See also the early work of Lieb-Simon [6] on molecules,

Received by the editors May 7, 1990.

1980 Mathematics Subject Classification (1985 Revision). Primary 81G45, $81 \mathrm{C} 15$.

The first author is partially supported by a NSF grant at Princeton University. 
the exposition of Hughes' proof in [10], and a different, shorter proof in [11].)

The argument of [1] begins with a pointwise inequality of Lieb [4] of the form

$$
\frac{1}{2} \sum_{i \neq j} \frac{1}{\left|x_{i}-x_{j}\right|} \geq \sum_{k=1}^{N} \widetilde{W}\left(x_{k}\right)-C .
$$

This implies

$$
H_{Z, N} \geq \sum_{k=1}^{N}\left(-\Delta_{x_{k}}-\frac{Z}{\left|x_{k}\right|}+\widetilde{W}\left(x_{k}\right)\right)-C
$$

and the right-hand side reduces by separation of variables to a problem in ordinary differential equations (ODEs). Our theorem is also proved by reduction to ODEs, but in place of (1) we need an estimate that takes account of the correlations between electrons on a length scale of $Z^{-2 / 3}$. Also, in studying ODEs, we need to replace the standard WKB approximation by a more precise approximation that contains the leading correction to WKB. Finally, to combine results for ODEs into estimates for spherically symmetric Schrödinger operators on $\mathbf{R}^{3}$, we must use a bit of analytic number theory. These are the main points in our proof. A brief sketch of our proof is as follows.

Let $\rho(x), W(x)$ be the Thomas-Fermi density and its screened Coulomb potential. Thus

$$
W(x)=-\frac{Z}{|x|}+\int_{\mathbf{R}^{3}} \frac{\rho(y) d y}{|x-y|} \leq 0
$$

and $6 \pi^{2} \rho(x)=|W(x)|^{3 / 2}$. See [5].

Let $E_{k}, \psi_{k}(x), 1 \leq k \leq N$, be the eigenvalues and (normalized) eigenfunctions of the one-electron Hamiltonian $-\Delta+W(x)$ on $L^{2}\left(\mathbf{R}^{3}\right)$. From the $\psi_{k}$ we make the Hartree-Fock wave function

$$
\psi_{h f}\left(x_{1}, \ldots, x_{N}\right)=\frac{1}{\sqrt{N !}} \sum_{\sigma} \psi_{\sigma 1}\left(x_{1}\right) \cdots \psi_{\sigma N}\left(x_{N}\right) \operatorname{sgn} \sigma .
$$

Its energy $E_{h f}=\left\langle H_{N, Z} \psi_{h f}, \psi_{h f}\right\rangle$ should approximate $E(Z)$ with excellent precision, as every chemist and physicist knows. We make a rigorous comparison by proving the following estimate. 


\section{Lemma 1.}

$$
\begin{aligned}
E(Z) \geq & \sum_{k=1}^{N} E_{k}-\frac{1}{2} \int_{\mathbf{R}^{3} \times \mathbf{R}^{3}} \frac{\rho(x) d x \rho(y) d y}{|x-y|} \\
& -c_{D} \int_{\mathbf{R}^{3}} \rho^{4 / 3}(x) d x-C Z^{(5 / 3)-a} \\
\equiv & E_{L B},
\end{aligned}
$$

where $c_{D}$ is Dirac's constant, and $a>0$.

This lemma is our substitute for inequality (1), and is a nontrivial step. Comparing the definition of $E_{L B}$ with the textbook formula for $E_{h f}$, we obtain the formula

$$
\begin{aligned}
& E_{h f}-E_{L B}=\frac{1}{2} \int_{\mathbf{R}^{3} \times \mathbf{R}^{3}} \frac{\left[\rho_{\#}(x)-\rho(x)\right]\left[\rho_{\#}(y)-\rho(y)\right]}{|x-y|} d x d y \\
& \quad+c_{D} \int_{\mathbf{R}^{3}} \rho^{4 / 3} d x-\frac{1}{2} \int_{\mathbf{R}^{3} \times \mathbf{R}^{3}} \frac{|S(x, y)|^{2}}{|x-y|} d x d y+C Z^{(5 / 3)-a}
\end{aligned}
$$

with

$$
\rho_{\sharp}(x)=\text { particle density }=\sum_{k=1}^{N}\left|\psi_{k}(x)\right|^{2}
$$

and

$$
S(x, y)=\text { correlation function }=\sum_{k=1}^{N} \psi_{k}(x) \bar{\psi}_{k}(y) .
$$

Next, we compare $\rho_{\#}(x)$ and $S(x, y)$ with their semiclassical approximations, and derive the following results.

\section{Lemma 2.}

$$
\int_{\mathbf{R}^{3} \times \mathbf{R}^{3}} \frac{\left[\rho_{\#}(x)-\rho(x)\right]\left[\rho_{\#}(y)-\rho(y)\right]}{|x-y|} d x d y \leq C Z^{(5 / 3)-a} \text {. }
$$

\section{Lemma 3.}

$$
\int_{\mathbf{R}^{3} \times \mathbf{R}^{3}} \frac{|S(x, y)|^{2}}{|x-y|} d x d y \geq c_{D} \int_{\mathbf{R}^{3}} \rho^{4 / 3}(x) d x-C Z^{(5 / 3)-a} \text {. }
$$

Putting Lemmas 2 and 3 into equation (2), we see that $E_{h f}-$ $E_{L B} \leq C^{\prime} Z^{(5 / 3)-a}$. Therefore Lemma 1 yields the chain of inequalities

$$
E_{h f} \geq E(Z) \geq E_{L B} \geq E_{h f}-C^{\prime} Z^{(5 / 3)-a}
$$

so that the three energies $E_{h f}, E(Z)$ and $E_{L B}$ all differ by $O\left(Z^{(5 / 3)-a}\right)$. Hence, the proof of our theorem reduces to the computation of $E_{L B}$, which evidently amounts to computing $\sum_{k=1}^{N} E_{k}$. 


\section{Lemma 4.}

$$
\begin{aligned}
\sum_{k=1}^{N} E_{k}= & -\frac{1}{15 \pi^{2}} \int_{\mathbf{R}^{3}}|W(x)|^{5 / 2} d x+\frac{1}{8} Z^{2} \\
& +\frac{1}{48 \pi^{2}} \int_{\mathbf{R}^{3}}|W(x)|^{1 / 2} \Delta W(x) d x+O\left(Z^{(5 / 3)-a}\right) .
\end{aligned}
$$

The Thomas-Fermi equations $-\Delta W=4 \pi \rho$ and $|W|^{3 / 2}=$ $6 \pi^{2} \rho$ let us rewrite the right-hand side here in terms of $\rho$. Substituting the result of Lemma 4 into the definition of $E_{L B}$, we obtain Schwinger's formula for $E(Z)$ with an error $O\left(Z^{(5 / 3)-a}\right)$. The constants $c_{0}$ and $c_{2}$ are specified by the equations

$$
\text { Thomas-Fermi Energy }=-c_{0} Z^{7 / 3}
$$

and

$$
c_{D} \int_{\mathbf{R}^{3}} \rho^{4 / 3}(x) d x=c_{2} Z^{5 / 3} .
$$

We close with a few comments on the proofs of Lemmas 2, 3, and 4. First of all, Lemma 2 is a weak substitute for a refined density formula, which is asserted in the physics literature but is easily seen to be false. Thus, we are fortunate in needing only the weak substitute. Lemma 3 is rather easy, since the main terms have size $Z^{5 / 3}$, while the error term is $O\left(Z^{(5 / 3)-a}\right)$. Lemma 4 requires the leading correction to the WKB approximation. We omit the precise statement, and just give the main formula. If $V(x)$ is a large slowly varying potential on the line, then $E$ closely approximates an eigenvalue of $-\left(d^{2} / d x^{2}\right)+V(x)$ when

$$
\begin{aligned}
& \int_{E \geq V(x)}(E-V(x))^{1 / 2} d x \\
& +\frac{1}{48} \lim _{\delta \rightarrow 0}\left(\int_{E-\delta>V(x)}(E-V(x))^{-3 / 2} V^{\prime \prime}(x) d x-\delta^{-1 / 2} G(E)\right) \\
& \quad=\pi\left(k+\frac{1}{2}\right)
\end{aligned}
$$

with $k$ an integer and $G(E)$ uniquely specified by requiring the finiteness of the limit. We use (3) to control eigenvalues in the range $\left[-Z^{(5 / 3)-\varepsilon}, 0\right]$. Eigenvalues below $-Z^{(5 / 3)-\varepsilon}$ come from the region near the origin where $W(x) \sim-(Z /|x|)+C$ and hence may be controlled by comparison with the elementary operator $-\Delta-(Z /|x|)+C$. Finally, we repeat that some simple analytic 
number theory enters when we sum over angular momenta to derive Lemma 4 from results on ODEs. The issues are closely related to the standard number-theoretic problem of counting the lattice points in a ball. In retrospect, this should not be surprising, since the lattice point problem clearly deals with the accuracy of the semiclassical eigenvalue count. The number-theoretic part of our proof requires a condition on the potential that plays the role of the nonvanishing curvature of the ball. Specifically, if $\phi(w)=\int_{0}^{\infty}\left(-\left(w^{2} / r^{2}\right)-W(x)\right)_{+}^{1 / 2} d r$, then we require that $\phi^{\prime \prime}(w)$ vanish at most to finite order for any $w$ in $\left(0, \max _{r} r|W(r)|^{1 / 2}\right)$.

This condition holds for the screened Thomas-Fermi potential, but fails for highly degenerate eigenvalue problems such as the harmonic oscillator or the hydrogen atom. Thus elementary analytic number theory explains the following paradox. Schwinger's eigenvalue formulas [7] are derived from a careful study of the harmonic oscillator and the hydrogen atom, yet these formulas are false for the harmonic oscillator and hydrogen atom. Nevertheless, they are correct for generic radial potentials, including the one we need. Understanding the class of nonradial potentials for which Schwinger's formulas hold is a hard, interesting problem.

Our results show that the ground state of an atom has twoelectron correlations on a length scale of $Z^{-2 / 3}$ equal to those of the Hartree-Fock wave function, modulo small corrections.

Finally, as an application of our results, we point out the following. In $[2,3,8]$, bounds for the excess charge and ionization energy for large atoms were obtained. These bounds were given as a function of a parameter $b$, between $\frac{1}{3}$ and $\frac{2}{3}$. The optimal bounds correspond to the value $\frac{2}{3}$. From the work of Hughes, Siedentop, and Weikard, it could be seen that one could take $b=\frac{3}{8}$. Our present work shows that one can take $b=\frac{2}{3}$.

We are grateful to R. Weikard for useful discussions.

\section{REFERENCES}

1. W. Hughes, Adv. Math. (to appear).

2. C. Fefferman and L. Seco, An upper bound for the number of electrons in a large ion, Proc. Nat. Acad. Sci. USA 88 (10) (1989), 3464-3465.

3. _ Asymptotic neutrality of large ions, Comm. Math. Phys. 128 (1990), 109-130.

4. E. H. Lieb, A lower bound for coulomb energies, Phys. Let. 70A (1979), 444-446. 
5. __ Thomas-Fermi and related theories of atoms and molecules, Rev. Modern Phys. 53 (4) (1981).

6. E. Leib and B. Simon, Thomas-Fermi theory of atoms, molecules and solids, Adv. Math. 23 (1977), 22-116.

7. J. Schwinger, Thomas-Fermi model: The second correction, Physical Review A24, vol. 5, Amer. Inst. Physics, New York, 1981, pp. 2353-2361.

8. L. Seco, I. M. Sigal, and J. P. Solovej, Bound on the ionization energy of large atoms, Comm. Math. Phys. (to appear).

9. H. Siedentop and R. Weikard, On the leading energy correction for the statistical model of the atom: Interacting case, Comm. Math. Phys. 112 (1987), 471-490.

10. __ On the leading correction of the Thomas-Fermi model: Lower bound, Invent. Math. 97 (1989), 159-193.

11. _ A lower bound of Scott type by a new microlocalization technique (to appear).

Department of Mathematics, Princeton University, Princeton, New JERSEY

Department of Mathematics, California Institute of Technology, Pasadena, California 